\title{
Expression of human protection of telomere 1 correlates with telomere length and radiosensitivity in the human laryngeal cancer Hep-2 cell line
}

\author{
HAN LEI $^{1 *}$, DALI FENG ${ }^{1 *}$, FUXIANG ZHOU ${ }^{1,2}$, HUI XU $^{1}$, TIAN TANG ${ }^{1}$, \\ HAIJUN $\mathrm{YU}^{1,2}$, CONGHUA XIE ${ }^{1,2}$ and YUNFENG ZHOU ${ }^{1,2}$ \\ ${ }^{1}$ Hubei Cancer Clinical Study Center, Hubei Key Laboratory of Tumor Biological Behaviors; \\ ${ }^{2}$ Department of Radiation Oncology and Medical Oncology, Zhongnan Hospital, Wuhan University, Wuhan, Hubei, P.R. China
}

Received October 7, 2014; Accepted May 15, 2015

DOI: $10.3892 / 01.2015 .3332$

\begin{abstract}
The close association between telomere length and radiosensitivity has been established by several studies. There is also a hypothesis that telomere length may be regulated by human protection of telomere 1 (hPOT1) in human carcinoma cells. In the present study, the hPOT1 level between the radioresistant Hep-2R cells and the wild-type were compared, and the results showed that the hPOT1 gene was upregulated in the radioresistant Hep-2R cell lines compared with the wild-type. This suggested that the expression level of hPOT1 correlates with radiosensitivity. Additionally, an hPOT1-directed short hairpin (sh)RNA plasmid was constructed and transferred into the Hep-2R cells, which lead to telomere shortening, an increase in apoptosis and markedly decreased growth of the RNAi-Hep-2R cell line. These results demonstrate that hPOT1-directed shRNAs are associated with telomere length and radiosensitivity, and maybe a potent sensitizer for laryngeal cancer radiotherapy.
\end{abstract}

\section{Introduction}

Radiation therapy is a well-established method that has been used to treat $>70 \%$ of patients with cancer. Although radiotherapy technology is continuously improving, the treatment effect of patients with carcinoma who receive radiotherapy is unsatisfactory, mainly due to radioresistance (1). Our previous study indicated that a significant negative correlation was present between the length of the telomeres and radiosensitivity in the same tissue-derived cell lines (2). Moreover, the

Correspondence to: Dr Fuxiang Zhou, Department of Radiation Oncology and Medical Oncology, Zhongnan Hospital, Wuhan University, 169 Donghu Road, Wuhan, Hubei 430071, P.R. China

E-mail: happyzhoufx@sina.com

${ }^{*}$ Contributed equally

Key words: telomere, radiosensitivity, hPOT1, telomere dysfunction gene expression profiles of a radiosensitive-radioresistant cell line model were compared on two complementary (c)DNA microarrays containing 14,000 human genes, which suggested that the expression of protection of telomere 1 (POT1) was significant upregulated by 3.348-fold in the radioresistant Hep2 cancer cells compared with the radiosensitive Hep2 cells (3).

POT1, a 3' single-stranded overhang telomeric DNA binding protein, has been identified in fission yeast and humans (4). A previous study indicated that each Pot1 protein binds to a single telomeric repeat and coats the whole single-stranded overhang of the telomere. Additionally, subsequent genetic and biochemical studies identified a role for POT1 in the maintenance of telomere length and in telomere capping (4). The aforementioned data indicates that there may be a correlation among POT1, telomere length and radiosensitivity in human cancer cells.

Despite these extensive studies into the biology of POT1, the role of POT1 expression in radiosensitivity and telomere function regulation in human cancer cells is currently unknown. In the present study, the association between hPOT1 and telomere length and radiosensitivity was investigated in the laryngeal cancer Hep-2 cell line.

\section{Materials and methods}

Cell culture. Human larynx squamous carcinoma Hep-2 cells (Cancer Research Center of Wuhan University, Wuhan, Hubei, China) were cultured in RPMI-1640 medium supplemented with $10 \%$ fetal bovine serum and incubated at $37^{\circ} \mathrm{C}$ in a humidified atmosphere containing $5 \% \mathrm{CO}_{2}$. For transfections, the cells were allowed to grow overnight to reach $80-90 \%$ confluence.

Isolation of radioresistant variant cell line. The radioresistant human larynx squamous carcinoma cell line (Hep-2R) was isolated by repeated radiation exposure, as previously described (5). Briefly, exponentially growing Hep-2 cells in $50-\mathrm{cm}^{2}$ culture flasks were exposed to a dose of $637 \mathrm{cGy}$ ${ }^{60}$ Co rays (field size, $20 \times 20 \mathrm{~cm}$; source-skin distance, $80 \mathrm{~cm}$; $61.3 \mathrm{cGy} / \mathrm{min}$ ) at room temperature, and the culture medium was changed following irradiation. When the irradiated cells reached the end of the exponential growth phase, they were trypsinized and appropriate numbers of cells were plated in 
$50-\mathrm{cm}^{2}$ culture flasks. Re-irradiation was performed at 1 day post-seeding. For the cell line designated as Hep-2R, the cells were isolated by repeating this procedure 12 times, and have already been maintained for $>50$ passages.

hPOT1 detection by reverse transcription polymerase chain reaction $(R T-P C R)$. The plasmid pshRNA-hPOT1 was transfected into the Hep-2R cells using reagent (Invitrogen Life Technologies, Carlsbad, CA, USA) according to the manufacturer's instructions. Total RNA was extracted from the parent Hep-2 cells with TRIzol reagent (Invitrogen Life Technologies) according to the manufacturer's instructions. The first strand of cDNA was obtained using a Revert Aid First Strand cDNA Synthesis kit (Fermentas, Thermo Fisher Scientific, Pittsburgh, PA, USA). For the quantitative analysis of hPOT1 mRNA, $\beta 2$-microglobulin was used as an internal control. The cycling conditions for the hPOT1 cDNA included preincubation for $5 \mathrm{~min}$ at $94^{\circ} \mathrm{C}$, followed 30 by cycles of $30 \mathrm{sec}$ at $94^{\circ} \mathrm{C}, 40 \mathrm{sec}$ at $58^{\circ} \mathrm{C}, 60 \mathrm{sec}$ at $72^{\circ} \mathrm{C}$ and a final extension for $5 \mathrm{~min}$ at $72^{\circ} \mathrm{C}$. The PCR products were identified using electrophoresis on $2 \%$ agarose gels and stained with ethidium bromide $(0.5 \mu \mathrm{g} / \mathrm{ml})$. Gel images were obtained and the densities of the PCR products were quantified with Bio-profil Bio-1D gel analysis software (Vilber Lourmat Co., Torcy, France).

hPOT1 detection by western blotting. The Hep-2 cells were transferred to ice-cold RIPA buffer (Life Technologies, Gaithersburg, MD, USA) containing $0.1 \mathrm{~mol} / 1 \mathrm{NaCl}$, $0.05 \mathrm{~mol} / \mathrm{l}$ Tris-HCl (pH 7.6), $0.001 \mathrm{~mol} / \mathrm{l}$ EDTA (pH 8.0), $0.1 \%$ Tween-20, aprotinin $(1 \mu \mathrm{g} / \mathrm{ml})$ and PMSF $(100 \mu \mathrm{g} / \mathrm{ml})$, and homogenized on ice. The homogenate was centrifuged at $10,000 \mathrm{x}$ g for $10 \mathrm{~min}$, and the supernatant was stored at $4^{\circ} \mathrm{C}$. Total protein concentrations were determined using the bicinchoninic acid protein assay kit (Biyuntian Biotech Co., Ltd., Shanghai, China). Samples were separated by electrophoresis on $7.5-12 \%$ polyacrylamide gels. Separated proteins were transferred onto polyvinylidene difluoride membranes at $30 \mathrm{~V}$ for $12 \mathrm{~h}$. The membrane was blocked with $5 \%$ dried, skimmed milk in Tris-buffered saline (TBS) buffer [50 mM Tris- $\mathrm{HCl}(\mathrm{pH} 7.5)$ with $150 \mathrm{mM} \mathrm{NaCl}$ containing $0.1 \%$ Tween-20 (TBST) for $1 \mathrm{~h}$ at room temperature. Blots were probed with specific polyclonal mouse anti-human antibodies (1:500; Affiniti Research, Devon, UK). Subsequent to being washed with TBST, the membranes were incubated for $1 \mathrm{~h}$ at room temperature with horseradish peroxidase-conjugated anti-mouse secondary antibody (1:2,500; Zhongshan Biotechnology, Beijing, China). Negative controls did not contain primary antibody. $\alpha$-tubulin was chosen as a loading control. The optical densities of the specific bands were scanned and measured by image analysis software (HPIAS 2000; Tongji Qianping Company, Wuhan, China).

Measurement of telomere length. Mean telomere length was determined by terminal restriction fragment (TRF) analysis using the Telo TAGGG Telomere Length Assay kit (Roche Diagnostics, Basel, Switzerland). According to the manufacturer's instructions, isolated DNA $(4 \mu \mathrm{g})$ was digested using a Hinf $\mathrm{I} / R s a \mathrm{I}$ enzyme mixture for $2 \mathrm{~h}$ at $37^{\circ} \mathrm{C}$. Digested DNA samples were then resolved by $0.7 \%$ agarose gel electrophoresis for 2-4 h at $5 \mathrm{~V} / \mathrm{cm}$. The gels were denatured, neutralized and transferred to a positively-charged nylon membrane, which was then hybridized with a biotinylated telomere probe. The mean TRF lengths were analyzed from a densitometric scan of the autoradiogram by the Bio-ID gel photograph system (Vilber Lourmat Co.) and calculated as described. The positive control DNA (control-DNA, low; and control-DNA, high) supplied with the kit is purified genomic DNA from immortal cell lines. The mean TRF length of these cell lines has been determined to be 3.9 and $10.2 \mathrm{~kb}$, respectively.

Short hairpin (sh)RNA preparation and plasmid construction. The plasmid pshRNA-hPOT1 was transfected into the Hep-2R cells using lipofectamine 2000 (Invitrogen Life Technologies). The shRNA was designed according to the hPOT1 sequence in the National Center for Biotechnology Information database (NM015450). The fitted target sequence was selected in the coding region without any homology to other genes by BLAST analysis. The shRNA contained a unique 19-nt double-stranded human hPOT1 sequence that is presented as an inverted complementary repeat and separated by a loop of a 9-nt spacer. The concrete shRNA sequence was shown as follows: 5'-GCCCCCATATCTAAGCAAA-3'. The sequence was cloned into the pGenesil plasmid. This new plasmid was termed pshRNA-hPOT1.

Clonogenic assay. The cells were trypsinized at $37^{\circ} \mathrm{C}$ for 5-10 min, and pipetted eight times to maintain the clumped cells as a single cell suspension. The single cell suspension was adjusted and seeded into $25-\mathrm{cm}^{2}$ flasks at various densities, based on the results of pre-experiments. Next, the cells were left to settle over night and then exposed to irradiation at room temperature, followed by immediate incubation at $37^{\circ} \mathrm{C}$, in $5 \% \mathrm{CO}_{2}$ for $14-20$ days. Subsequent to fixation and staining with $1 \% \mathrm{w} / \mathrm{v}$ crystal violet (Sigma-Aldrich, St. Louis, MO, USA) in dehydrated alcohol, colonies of $>50$ cells were scored as previously described (6). Surviving fractions (SF) were evaluated relative to $0 \mathrm{~Gy}$ radiation-treated controls.

TRF Southern blot analysis. A minimum of $3 \mu \mathrm{g}$ DNA was used for measurement of telomere length. DNA was extracted using a Gentra Puregene Cell Kit (Qiagen, Inc., Valencia, CA, USA). To evaluate DNA integrity, DNA samples (10 ng) were resolved on a $1 \%(\mathrm{wt} / \mathrm{vol})$ agarose gel at $200 \mathrm{~V}$ for $60 \mathrm{~min}$. DNA that appeared as a single compact crown-shaped band that migrated in parallel with the other samples on the gel was considered to be acceptable. A combination of the restriction enzymes HinfI and RsaI (Roche) was used to digest the genomic DNA. For TRF length analysis of human leukocyte telomere length, a $0.5 \%(\mathrm{wt} / \mathrm{vol})$ agarose gel was used to resolve the digested DNA with a combination of two commercially available DNA standards: A 1-kb ladder spanning 0.5-12 kb and a collection of $\lambda$ DNA fragments digested with Hind III spanning 1.25-23.1 kb (Invitrogen Life Technologies; catalog no. 15612-013). The telomere probe (Eurofins MWG Operon, Ebersberg, Germany) consisted of three oligonucleotide repeats of the sequence complementary to the canonical telomeric TTAGGG sequence, and was labeled at the 3 ' end with digoxigenin (DIG; DIG Oligonucleotide 3'-End Labeling Kit, Roche). After the hybridization and washing steps, the DIG-labeled probe was detected using an alkaline 
A

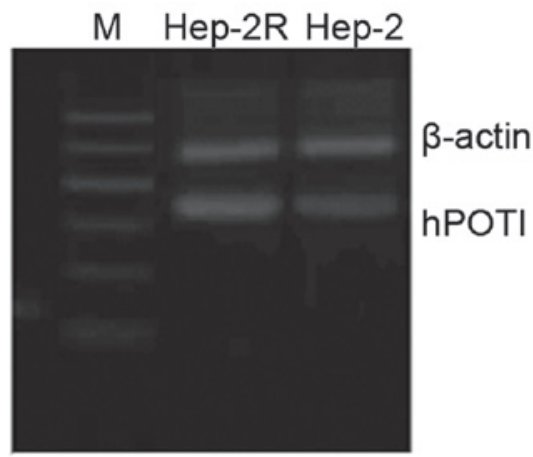

B

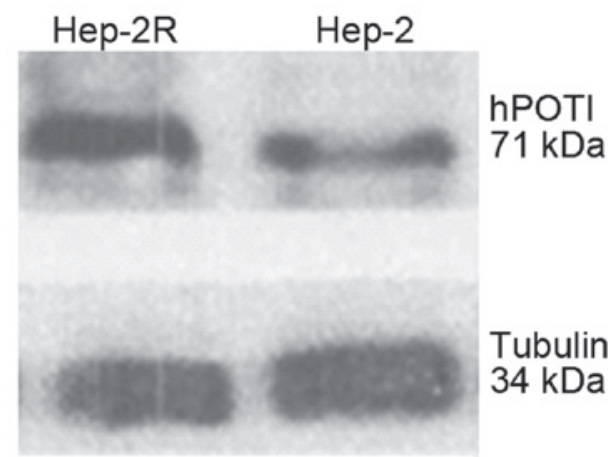

Figure 1. Human protection of telomere 1 (hPOT1) detection by reverse transcription polymerase chain reaction and western blotting between Hep-2 and Hep-2R cells. (A) The Hep-2R cells showed a significantly increased level of transcription of hPOT1 mRNA compared with the Hep-2 cells. (B) Western blotting showed a 71-kDa hPOT1 product when using the hPOT1 antibody in the Hep-2 and Hep-2R cell line.
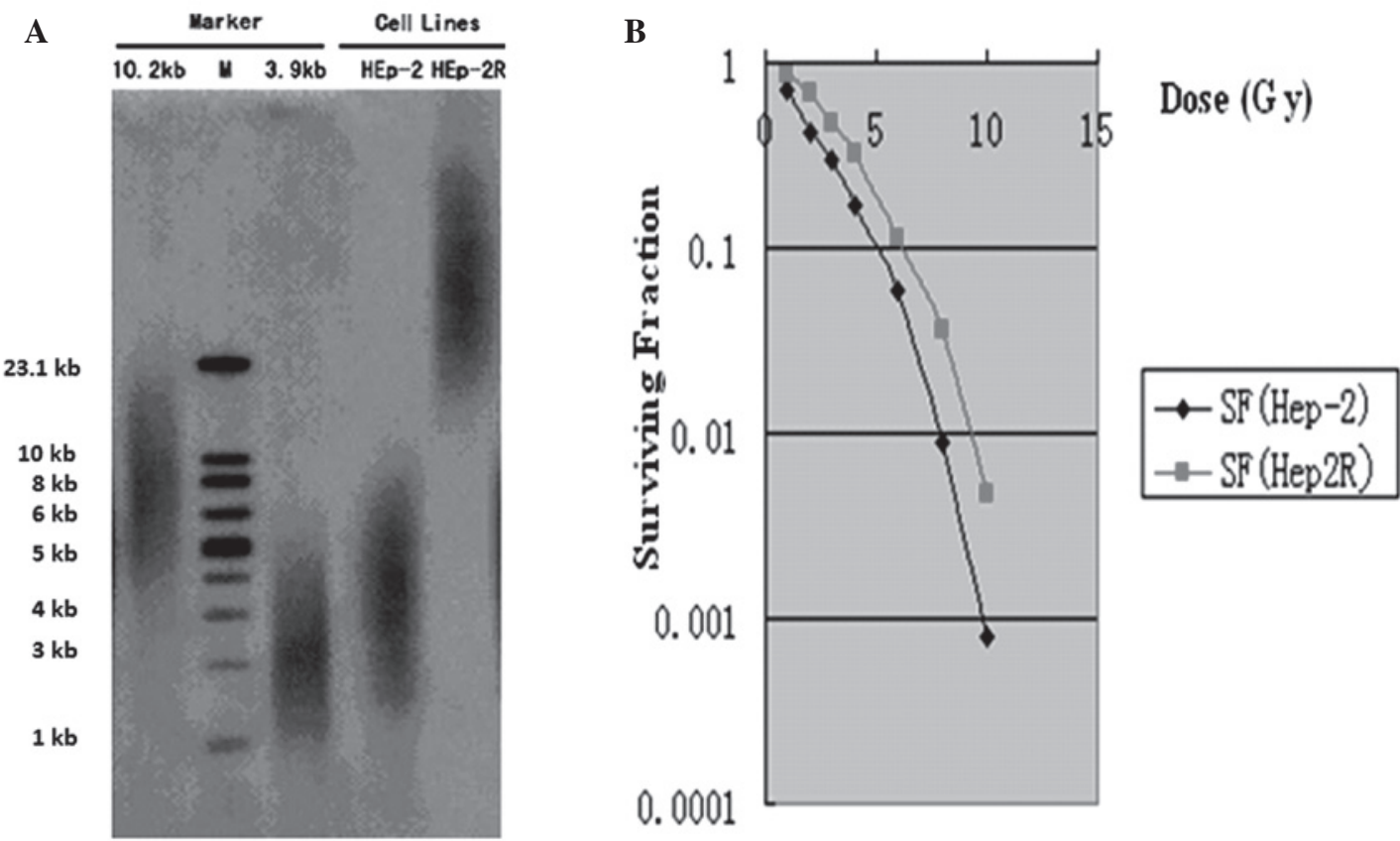

Figure 2. Correlation between telomere length and radiosensitivity. (A) Representative mean telomere length in Hep-2 and Hep-2R cells, as determined by restriction fragment analysis. (B) The clonogenic survival curves of the Hep-2 and Hep-2R cells. SF, surviving fraction.

phosphatase-conjugated sheep polyclonal anti-DIG antibody (1:500; catalog no. 11093274910; Roche) and visualized using chemiluminescence reagent (Lumigen APS-5; Lumigen, Southfield, MI, USA) and x-ray film (Amersham Hyperfilm ECL, GE Healthcare Life Sciences, catalog no. 28-906837; transparency film, Corporate Express Ltd., Birmingham, UK, catalog no. CEB00559; x-ray developer, Konica Minolta Inc., Tokyo, Japan). A premeasured grid was the superimposed over each lane, and the signal intensity within each box and corresponding molecular weight was used to calculate TRF length. A sample of known TRF length was also run in each Southern blot gel as an internal reference (7).

Assessment of apoptosis with the terminal deoxynucleotidyl transferase dUTP nick end labeling (TUNEL) assay. According to the manufacturer's instructions for the TUNEL assay (BD Biosciences, Heidelberg, Germany), the number of apoptotic cells was counted and then the tumor cells apoptotic index (AI) was calculated according to the following formula: $\mathrm{AI}=$ (number of apoptotic cells / number of total cancer cells) x 100 .

Cell growth analysis. A total of $1 \times 10^{5}$ Hep-2R and Hep-2 cells were seeded in each $25-\mathrm{cm}^{2}$ culture flask, respectively. The attached cells were trypsinized, and the cell numbers were determined by a hemocytometer at 24, 48 and $72 \mathrm{~h}$ post-seeding, respectively. The growth curve of these three cell lines was drawn for the aforementioned data.

Statistical analysis. All numerical experimental data were expressed as the mean \pm standard deviation, and statistical analyses of the data were performed with analysis of variance and interclass q test methods using SPSS11.0 statistical software (SPSS, Inc., Chicago, IL, USA). All P-values are based on two-side hypothesis testing, and $\mathrm{P}<0.05$ was used to indicate a statistically significant difference. 


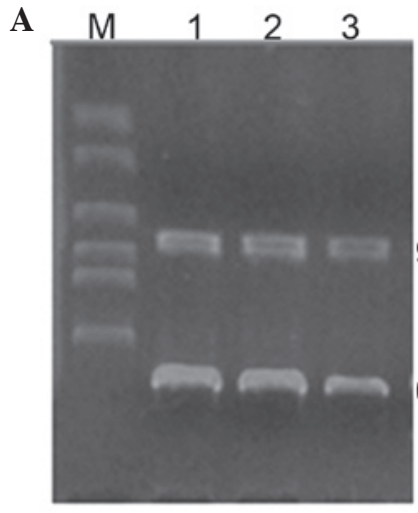

C

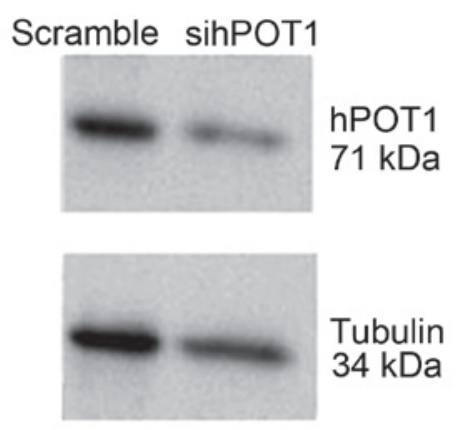

B

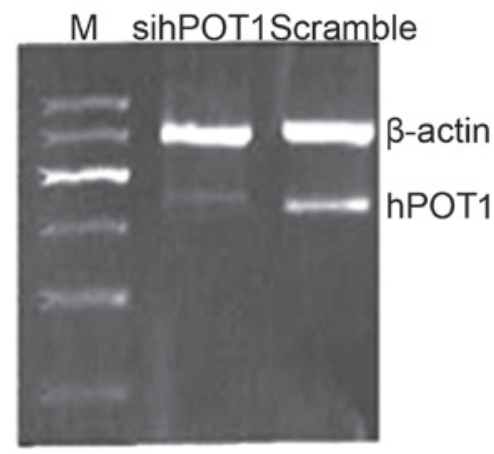

D

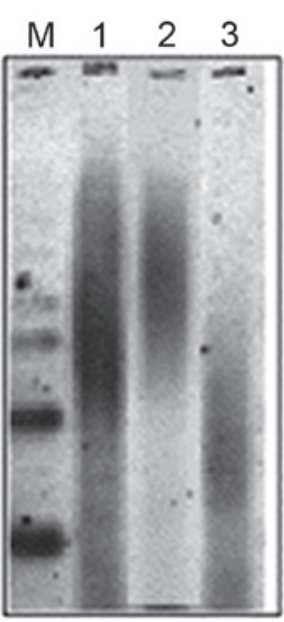

Figure 3. (A) Construction and identification of the recombinant plasmid with restriction enzyme. DNA fragments of 600 and 916 bp could be detected following treatment with SacI enzyme. (B) human protection of telomere 1 (hPOT1) detection following pshRNA-hPOT1 transfection, according to reverse transcription ploymerase chain reaction (RT-PCR) and western blotting. Representative RT-PCR products were separated on a 1\% agarose gel stained with ethidium bromide. (C) Western blotting analysis in Hep-2R cells expressing pshRNA-hPOT1 and control. (D) Measurement of telomere length by the TRF Southern blot analysis in the Hep-2R cells (lane 1), the control (lane 2) and the hPOT1-specific shRNA-expressing clones (lane 3). pshRNA, plasmid short hairpin RNA.
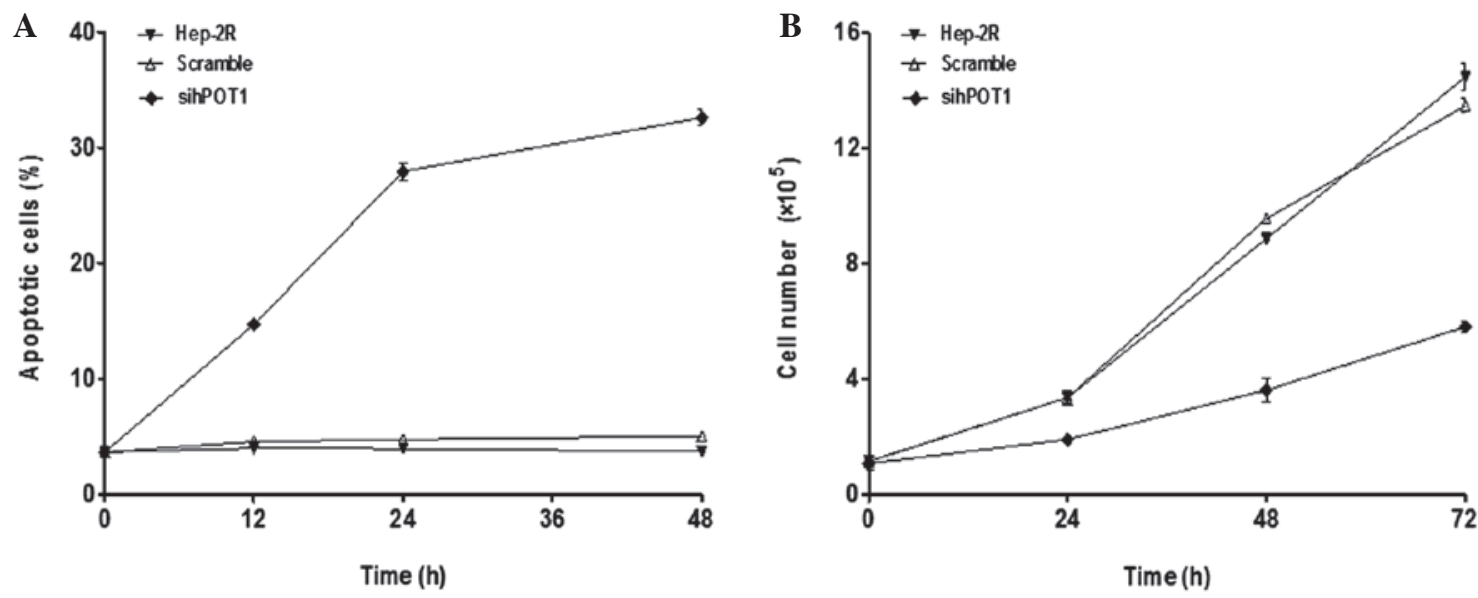

Figure 4. hPOT1-RNAi-induced apoptosis and senescence. (A) Terminal deoxynucleotidyl transferase dUTP nick end labeling assay of Hep-2R cells after expression with pshRNA-hPOT1. The apoptotic cells in the Hep-2R, control and RNAi groups were determined after 0, 12, 24 and $48 \mathrm{~h}$. (B) Growth curves of Hep-2R cells expressing pshRNA-hPOT1 and vector control cells. The difference in proliferative capacity between the Hep-2R and RNAi groups was significant. RNAi, RNA interference; pshRNA, plasmid short hairpin RNA; hPOT1, human protection of telomere 1.

\section{Results}

hPOT1 detection by RT-PCR and western blotting between Hep-2 and Hep-2R cells. As shown in Fig. 1A, the semiquantitative RT-PCR result suggested that the Hep-2R cells exhibited a significantly increased transcription level of hPOT1 mRNA compared with the Hep-2 cells. The quantity of hPOT1 mRNA in the Hep-2R cells was markedly increased by $38.12 \%$ compared with the Hep- 2 cells. Furthermore, hPOT1 protein expression in the Hep-2R 
cells was upregulated by $27.59 \%$ compared with the Hep-2 cells (Fig. 1B). The results indicated that in comparison to the Hep-2 cells, the radioresistant Hep-2R cells exhibited upregulation of the hPOT1 gene.

Correlation between telomere length and radiosensitivity in Hep-2 and Hep-2R cells. Fig. 2 presents the association between radiosensitivity and TRF length in the Hep-2 and Hep-2R cell lines, which were chosen for the present study in order to represent differing genetic backgrounds. The telomere lengths of the two cell lines were determined by restriction fragment analysis. It was determined that the Hep-2 and Hep-2R cell lines presented with an mean telomere length of $\sim 3.9$ and $10.2 \mathrm{~kb}$, respectively (Fig. 2A). Following normalization against the plating efficiency of the mock-irradiated cells, the survival curves of the cells were analyzed (Fig. 2B). The number of surviving fractions at $2 \mathrm{~Gy}(\mathrm{SF} 2)$ for the Hep-2R cells $(0.683)$ was significantly greater than that for the Hep-2 cells (0.415). Therefore, the mean telomere length of the radioresistant Hep- $2 \mathrm{R}$ cell line was greater than that of the radiosensitive Hep-2 cell line.

hPOT1 and telomere length detection following pshRNA-hPOT1 transfection. As shown in Fig. 3A, 600- and 916-bp DNA fragments could be detected following SacI enzyme treatment, suggesting that the recombinant plasmid was as expected. Furthermore, the coded sequence of the recombinant plasmid was sequenced (data not shown). The result indicated that the specific shRNA expression vector (named pshRNA-hPOT1) was successfully constructed, inhibiting hPOT1 gene expression. The quantity of hPOT1 mRNA was markedly deceased by $62.34 \%$ in the shRNA-hPOT1 transfectant compared with the scramble group (Fig. 3B). Fig. 3C showed that, in the RNA interference (RNAi) group, hPOT1 protein expression rates were lower than those of the same group in the scramble group $(44.52 \%)$, and there was significant difference $(\mathrm{P}<0.05)$. Since it was demonstrated that pshRNA-hPOT1 was effective to inhibit the expression of hPOT1, the effects of hPOT1 suppression on telomere length were then examined. The analysis of TRFs by Southern blotting demonstrated that the telomere length observed in the control group was $\sim 10 \mathrm{~kb}$, which was similar to that of the Hep-2R cells. By contrast, the telomere lengths in the RNAi group displayed significant telomere shortening, with lengths averaging 2-3 kb (Fig. 3D).

Inhibition of POT1 induces apoptosis and suppresses proliferative capacity. Telomere dysfunction can induce DNA damage response pathways, apoptosis and senescence. the present results showed that a significantly increased level of apoptosis was observed in the cells that were expressing the pshRNA-hPOT1, as assessed by TUNEL-labeling analysis (Fig. 4A), which was consistent with the results showing that hPOT1-RNAi could induce telomere dysfunction and apoptosis with the DNA damage response. The proliferative capacity prior to and following RNAi is shown in Fig. 4B. Hep-2R-RNAi cells showed a markedly decreased growth rate compared with the Hep-2R cells. In conclusion, the results suggested that cell proliferation was inhibited by transfection with pshRNA-hPOT1.

\section{Discussion}

Our results from earlier studies indicated that telomere length was inversely correlated with radiosensitivity in human carcinoma cells (8-10), which is consistent with other studies showing that there is an association between telomere length and radiosensitivity in human cells (11-13). Indeed, radiosensitive human cells show shorter telomeres than normal cells, and short telomeres increase cell radiosensitivity, so telomere has great appeal as a clinical target.

Telomeres are formed from repeats of the TTAGGG DNA sequence and associated proteins that cap the ends of linear chromosomes. The main function of these distinctive structures is to protect the chromosome ends and to prevent activation of the DNA damage response (14). Telomerase is a specialized enzyme that provides a telomere maintenance mechanism (15); in human cells, telomerase functions as a reverse transcriptase to add multiple copies of the GGTTAG motif to the end of the $\mathrm{G}$-strand of the telomere (15). The telomere can be regulated by proteins identified during the last decade that make up a complex called shelterin (16). This complex is composed of six proteins. The telomeric single-stranded overhangs have been indicated to be a vital component of the telomeric structure required for correct telomeric function. POT1, one of the shelterin complex proteins, is a single-stranded telomeric DNA-binding protein that has been identified in fission yeast and humans (17).

In the present study, the hPOT1 gene was upregulated in the radioresistant Hep-2R cell line compared with the wild-type Hep- 2 cell line. At the same time, the mean telomere length in the radioresistant Hep-2R cell line was greater than that in the radiosensitive Hep-2 cell line. Therefore, it was hypothesized that the expression of hPOT1 correlates with telomere length and radiosensitivity. In order to verify this hypothesis, pshRNA targeting hPOT1 was constructed. The experimental results indicated that telomere lengths in the RNAi group exhibited significant shortening, together with an increase in cell apoptosis and a decreased proliferative capacity, with downregulated hPOT1. Overall, these findings suggest that telomere length could be regulated by hPOT1 in human carcinoma cells, which is associated with radiosensitivity.

One potential role for the hPOT1 protein is the binding of telomere ends and the stabilization of the telomeres $(18,19)$. This may allow telomerase greater access to the telomere and lead to an increase in the length of the telomere; this role would be consistent with our results showing telomerase-dependent telomere elongation by hPOT1 (20). hPOT1 may also actively recruit the telomerase complex to the end of the telomere (16). Based on the aforementioned data, the inhibition of hPOT1 protein may induce telomere shortening due to reduced telomerase recruitment. hPOT1 may be a potential therapeutic target of telomerase-positive tumors.

Furthermore, hPOT1 is required at the telomeric single-stranded overhangs in order to maintain the normal structure, protect against apoptosis, and prevent the instability and senescence of the chromosome (21-23). The erosion of the single-stranded overhangs in hPOT1-deficient cells immediately causes telomere dysfunction. Telomere dysfunction can induce DNA damage response pathways, apoptosis and senescence. The conclusion was confirmed by the present study results. An increase in apoptosis and a marked decrease in 
growth were observed in the RNAi-Hep-2R cell lines, which was consistent with previous results showing that hPOT1-RNAi could induce telomere dysfunction and apoptosis with the DNA damage response (24). Further investigations are required to clarify the mechanisms involved in detail.

In conclusion, the elucidation of telomere regulation may improve the therapeutic regulation of radiotherapy in human tumor cells. Based on a review of the existing literature, it can be concluded that hPOT1 may correlate with telomere length and radiosensitivity, and will be a good target for cancer radiotherapy.

\section{Acknowledgements}

This study was supported by the National Natural Science Foundation of China (grant no. 30770643).

\section{References}

1. Buczek M, Escudier B, Bartnik E, Szczylik C and Czarnecka A: Resistance to tyrosine kinase inhibitors in clear cell renal cell carcinoma: From the patient's bed to molecular mechanisms. Biochim Biophys Acta 1845: 31-41, 2014.

2. Tang T, Zhou FX, Lei H, Yu HJ, Xie CH, et al: Increased expression of telomere-related proteins correlates with resistance to radiation in human laryngeal cancer cell lines. Oncol Rep 21: 1505-1509, 2009.

3. Zhou FX, Xiong J, Luo ZG, Dai J, Yu HJ, et al: cDNA expression analysis of a human radiosensitive-radioresistant cell line mode identifies telomere function as a hallmark of radioresistance. Radiat Res 174: 550-557, 2010.

4. Lei M, Podell ER and Cech TR: Structure of human POT1 bound to telomeric single-stranded DNA provides a model for chromosome end-protection. Nat Struct Mol Biol 11: 1223-1229, 2004

5. Wu F, Hu Y, Long J, Zhou YJ, Zhong YH, Liao ZK, Liu SQ, Zhou FX, Zhou YF and Xie CH: Cytotoxicity and radiosensitization effect of TRA-8 on radioresistant human larynx squamous carcinoma cells. Oncol Rep 21: 461-465, 2009.

6. Franken NA, Rodermond HM, Stap J, Haveman J and van Bree C: Clonogenic assay of cells in vitro. Nat Protoc 1: 2315-2319, 2006.

7. Kimura M, Stone RC, Hunt SC, Skurnick J, Lu X, Cao X, Harley CB and Aviv A: Measurement of telomere length by the Southern blot analysis of terminal restriction fragment lengths. Nat Protoc 5: 1596-1607, 2010.

8. Zhong YH, Liao ZK, Zhou FX, Xie CH, Xiao CY, et al: Telomere length inversely correlates with radiosensitivity in human carcinoma cells with the same tissue background. Biochem Biophys Res Commun 367: 84-89, 2008.

9. Zhou FX,Liao ZK, Dai J, Xiong J, Xie CH, et al: Radiosensitization effect of zidovudin on human malignant glioma cells. Biochem Biophys Res Commun 354: 351-356, 2007.
10. Neuhof D, Ruess A, Wenz F and Weber KJ: Induction of telomerase activity by irradiation in human lymphoblasts. Radiat Res 155: 693-697, 2001

11. Goytisolo FA, Samper E, Edmonson S, Taccioli GE and Blasco MA: The absence of the DNA-dependent protein kinase catalytic subunit in mice results in anaphase bridges and in increased telomeric fusions with normal telomere length and G-strand overhang. Mol Cell Biol 21: 3642-3651, 2001.

12. McIlrath J, Bouffler SD, Samper E, Cuthbert A, Wojcik A, et al: Telomere length abnormalities in mammalian radiosensitive cells. Cancer Res 61: 912-915, 2001.

13. Wong KK, Chang S, Weiler SR, Ganesan S, Chaudhuri J, et al: Telomere dysfunction impairs DNA repair and enhances sensitivity to ionizing radiation. Nat Genet 26: 85-88, 2000.

14. Masutomi K, Yu EY, Khurts S, Ben-Porath I, Currier JL, et al: Telomerase maintains telomere structure in normal human cells. Cell 114: 241-253, 2003.

15. Wang F, Podell ER, Zaug AJ, Yang Y, Baciu P, et al: The POT1-TPP1 telomere complex is a telomerase processivity factor. Nature 445: 506-510, 2007.

16. Hockemeyer D1, Sfeir AJ, Shay JW, Wright WE and de Lange T: POT1 protects telomeres from a transient DNA damage response and determines how human chromosomes end. EMBO J 24: 2667-2678, 2005

17. Zhu XD, Niedernhofer L, Kuster B, Mann M, Hoeijmakers JH and de Lange T: ERCC1/XPF removes the 3' overhang from uncapped telomeres and represses formation of telomeric DNA-containing double minute chromosomes. Mol Cell 12: 1489-1498, 2003.

18. Lacroix L, Liénard H, Labourier E, Djavaheri-Mergny M, Lacoste $\mathrm{J}$, et al: Identification of two human nuclear proteins that recognize the cytosine-rich strand of human telomeres in vitro. Nucleic Acids Res 28: 1564-1575, 2000

19. Gomez D1, Paterski R, Lemarteleur T, Shin-Ya K, Mergny JL and Riou JF: Interaction of telomestatin with the telomeric single-strand overhang. J Biol Chem 279: 41487-41494, 2004.

20. Liu D, O'Connor MS, Qin J and Songyang Z: Telosome, a mammalian telomere-associated complex formed by multiple telomeric proteins. J Biol Chem 279: 51338-51342, 2004.

21. He H, Multani AS, Cosme-Blanco W, Tahara H, Ma J, et al: POT1b protects telomeres from end-to-end chromosomal fusions and aberrant homologous recombination. EMBO J 25: 5180-5190, 2006

22. Takai H, Smogorzewska A and de Lange T: DNA damage foci at dysfunctional telomeres. Curr Biol 13: 1549-1556, 2003.

23. Blasco MA: The epigenetic regulation of mammalian telomeres. Nat Rev Genet 8: 299-309, 2007.

24. d'Adda di Fagagna F1, Reaper PM, Clay-Farrace L, Fiegler H, Carr P, Von Zglinicki T, Saretzki G, Carter NP and Jackson SP: A DNA damage checkpoint response in telomere-initiated senescence. Nature 426: 194-198, 2003. 